\title{
Charge order textures induced by non-linear lattice coupling in a half-doped manganite
}

\section{Ismail El Baggari ( $\square$ ie52@cornell.edu )}

Cornell University

\section{David Baek}

Cornell University

\section{Michael Zachman}

Cornell University

\section{Di Lu}

Stanford University

\section{Yasuyuki Hikita}

Denso Corporation

Harold Hwang

Stanford University https://orcid.org/0000-0002-9230-3214

Elizabeth Nowadnick

University of California, Merced

\section{Lena Kourkoutis}

Cornell University https://orcid.org/0000-0002-1303-1362

\section{Article}

Keywords: lattice coupling, manganite, local order

Posted Date: December 1st, 2020

DOI: https://doi.org/10.21203/rs.3.rs-103592/v1

License: (c) (1) This work is licensed under a Creative Commons Attribution 4.0 International License. Read Full License

Version of Record: A version of this preprint was published at Nature Communications on June 18th, 2021. See the published version at https://doi.org/10.1038/s41467-021-24026-7. 


\title{
Charge order textures induced by non-linear lattice coupling in a half-doped manganite
}

\author{
Ismail El Baggari, ${ }^{1, *}$ David J. Baek, ${ }^{2, a, *}$ Michael J. Zachman, ${ }^{3, b}$ Di Lu, ${ }^{4,5, c}$ Yasuyuki \\ Hikita, ${ }^{5,6}$ Harold Y. Hwang, ${ }^{5,6}$ Elizabeth A. Nowadnick, ${ }^{7}$ Lena F. Kourkoutis ${ }^{3,8}$ \\ ${ }^{1}$ Department of Physics, Cornell University, Ithaca, NY \\ ${ }^{2}$ School of Electrical and Computer Engineering, Cornell University, Ithaca, NY \\ ${ }^{3}$ School of Applied and Engineering Physics, Cornell University, Ithaca, NY \\ ${ }^{4}$ Department of Physics, Stanford University, Stanford, CA \\ ${ }^{5}$ Stanford Institute for Materials and Energy Sciences, \\ SLAC National Accelerator Laboratory, Menlo Park, CA \\ ${ }^{6}$ Department of Applied Physics, Stanford University, Stanford, CA \\ ${ }^{7}$ Department of Materials Science and Engineering, \\ University of California Merced, Merced CA \\ ${ }^{8}$ Kavli Institute at Cornell for Nanoscale Science, Cornell University, Ithaca, NY
}

${ }^{a}$ Current address: Intel Corp., Hillsboro, OR

${ }^{b}$ Current address: Center for Nanophase Materials Sciences, Oak Ridge National Laboratory, TN

${ }^{c}$ Current address: Materials Science and Engineering, Northwestern University, Evanston, IL

* These authors contributed equally to this work. 
The self-organization of strongly interacting electrons into superlattice structures underlies the properties of many quantum materials. How these electrons arrange within the superlattice dictates what symmetries are broken and what ground states are stabilized. Here we show that cryogenic scanning transmission electron microscopy enables direct mapping of local symmetries and order at the intra-unit-cell level in the model charge-ordered system $\mathrm{Nd}_{1 / 2} \mathrm{Sr}_{1 / 2} \mathrm{MnO}_{3}$. In addition to imaging the prototypical site-centered charge order, we discover the nanoscale coexistence of an exotic intermediate state which mixes site and bond order and breaks inversion symmetry. We further show that nonlinear coupling of distinct lattice modes controls the selection between competing ground states. The results demonstrate the importance of lattice coupling for understanding and manipulating the character of electronic self-organization and highlight a novel method for probing local order in a broad range of strongly correlated systems. 
Strong interactions between electrons and the atomic lattice often lead to their selforganization into ordered spatial patterns [1-4]. One well-known example is charge ordering, the spatial modulation of the electronic charge density which forms superlattices and governs the properties of many exotic materials, from oxides to transition-metal chalcogenides to charge-transfer salts [5-7]. In general, charge ordering is studied at scales larger than the superlattice, with a focus on the average periodicity of the charge modulation or the degree of long range order $[8,9]$. On the other hand, the microscopic arrangement at sub-unit-cell length scales, such as whether the electrons reside on the atomic sites or bonds (Fig. 1a and b), is significantly more challenging to measure, but dictates the symmetry of the system in addition to the mechanism underlying electronic order [10-12].

Perovskite $3 d$ transition-metal oxides are a class of materials in which charge order plays an especially important role, influencing antiferromagnetic order, high-temperature superconductivity, and colossal magnetoresistance $[5,13,14]$. The strong hybridization between the transition metal (site) and oxygen (bond) in these systems contributes to their rich, intricate electronic structure landscape [15]. In cuprates, for instance, recent bulk measurements suggest that the charge-ordered phase may contain a site-centered modulation in addition to the putative bond-centered $d$-wave modulation $[10,16]$. A similarly complex and fundamental debate concerns the half-doped manganites, an ideal playground for exploring coupled charge and orbital orders and phase competition [17]. The widely accepted model, proposed over 60 years ago, invokes a site-centered modulation in which electrons localize on manganese sites and form a zig-zag orbital order pattern which doubles the unit cell (Fig. 1c) [18-20]. An alternative bond-centered state has also been proposed [21]; in this case a charge modulation does not occur so the superlattice is generated from orbital ordering (Fig. 1d). Subsequent experimental studies, however, have not conclusively confirmed this scenario [20, 22, 23]. Theory further predicts an even more exotic intermediate state which mixes both site and bond characters, leading to the breaking of inversion symmetry through the formation of uncompensated electric dipoles [24, 25]. Determining if such a state exists or whether charge order corresponds to a pure site- or bond-centered state remains a fundamental challenge, with broad and urgent implications for other classes of materials.

Manganite compounds exhibit large interactions between the lattice and the electronic degrees of freedom, and so most experimental proposals for site- or bond-centered charge order models have relied on obtaining the average lattice distortions and crystal symmetry 
$[19,21]$. Here we employ a novel methodology for probing charge order, based on atomicresolution cryogenic scanning transmission electron microscopy (cryo-STEM) and the direct measurement of picoscale lattice displacements in real space. By mapping the displacement patterns within the charge order superlattice, this method enables us to visualize distinct, coexisting arrangements of charge order and, importantly, establish the existence of the intermediate phase. Absent the real space visualization, this phase would remain hidden, partly due to the challenge of disentangling nanoscale domains of pure bond/site order from genuine mixed states in bulk-averaged measurements. Going further, cryogenic STEM imaging and theoretical calculations reveal coupled secondary order parameters that govern the nature of the charge order phase. These atomic-scale visualizations not only advance our understanding of charge ordering through a novel lens but also reveal a tuning knob for manipulating electronic self-organization via lattice coupling.

The material we focus on is a $\mathrm{Nd}_{1 / 2} \mathrm{Sr}_{1 / 2} \mathrm{MnO}_{3}$ thin film which was grown using pulsed laser deposition on a (110)-oriented $\mathrm{SrTiO}_{3}$ substrate. The particular substrate orientation, which imparts in-plane anisotropy on the film, reproduces the electronic and structural transitions found in the bulk counterpart including the charge order transition [26-28]. The temperature dependence of the magnetization in this film matches that of previous reported epitaxial films. Figure 1e shows a projection HAADF-STEM image of the crystal along the [010] orientation (in the Pnma space group setting) below the charge order transition temperature. The atomically-resolved Nd/Sr columns appear brighter than the Mn columns because the contrast in HAADF scales strongly with the atomic number. Near the crystalline peaks in the Fourier transform amplitude (Fig. 1f), superlattice peaks (blue arrows) appear at low temperature, indicating the formation of a modulated structure. These are located at $\mathbf{Q}^{C O}=(1 / 2,0,0)$ as expected for charge ordering at half doping. The modulation is unidirectional, however, some regions of the sample exhibit bi-directional modulations. These might arise from the coexistence of small charge order domains (either in-plane or out-of-plane) within a single crystal twin or from the presence of crystalline twins that establish the direction of the charge order wavevector. By mapping both the charge order and the crystalline order parameters, we find that the orthogonal charge order domains are coupled to crystalline twins in the sample (Supplementary Fig. 3).

The exact intra-unit-cell arrangement within charge order superlattices is key to understanding their microscopic origins and interactions with other electronic phases. In the 
a

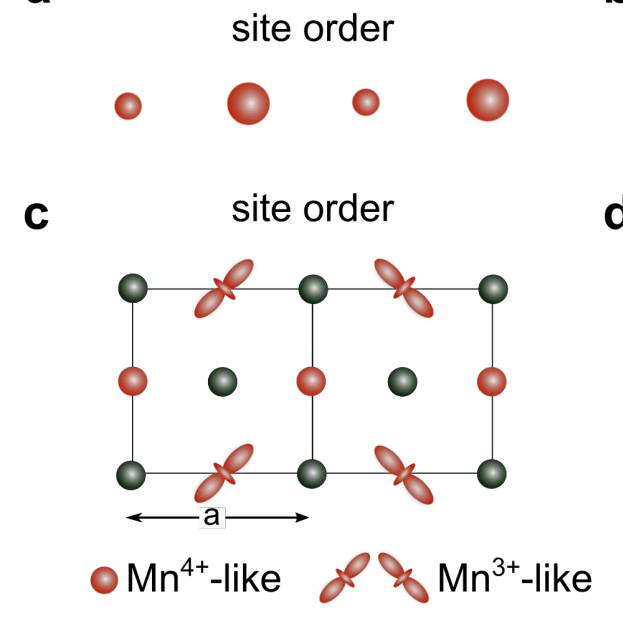

e

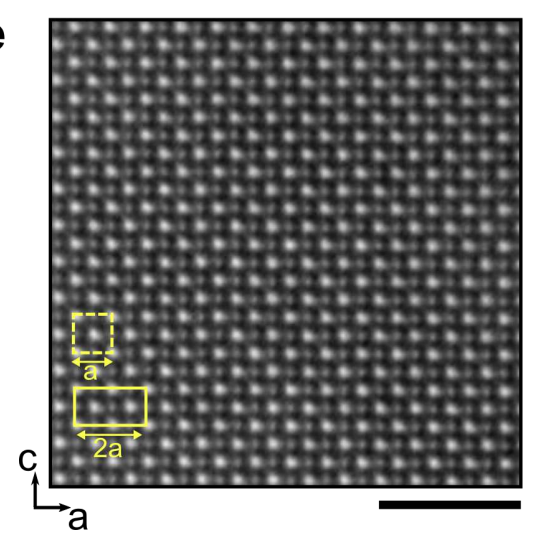

b

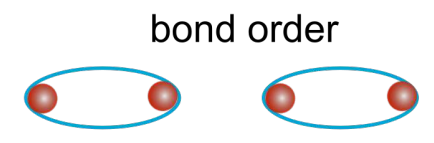

d bond order

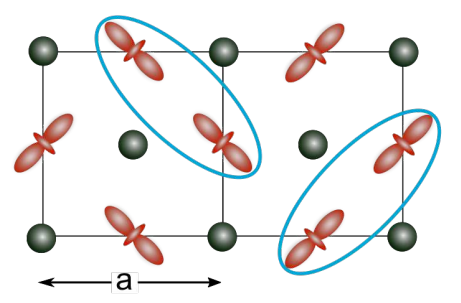

$\mathrm{ONd} / \mathrm{Sr}$ of $\mathrm{Mn}^{3.5+}$

f

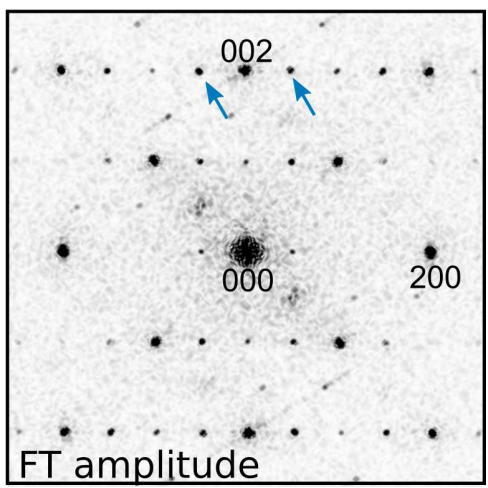

FIG. 1. Charge order supercell. a, Charge order modulation centered on sites (red circles). The size of the circle indicates the electronic density on the site. b, Charge order modulation centered on the bonds. Ellipses represent stronger, more electron-rich bonds. The sites remain equivalent in density. c, Prototypical site-centered charge and orbital order model in the half-doped manganites, including orbital order. d, Alternative bond-centered model in which all Mn sites have the same charge configuration $\left(\mathrm{Mn}^{3.5+}\right)$. In this case, the supercell is generated by the orbital order. e, Highangle annular dark-field (HAADF) scanning transmission electron microscopy (STEM) projection image at $93 \mathrm{~K}$, where we expect the system to be in the charge-ordered phase. Bright atomic columns represent $\mathrm{Nd} / \mathrm{Sr}$ sites and dark atomic columns represent $\mathrm{Mn}$ sites. The scale bar corresponds to $2 \mathrm{~nm}$. f, Fourier transform amplitude of the STEM image. At $93 \mathrm{~K}$, superlattice peaks (arrows) with $\mathbf{Q}^{C O}=(1 / 2,0,0)$ are evident, indicating the formation of a twofold superlattice in real space (box in E). 
majority of theoretical treatments of charge-ordered phases, the ground state is discussed in terms of pure electronic degrees of freedom. Site-centered charge order in half-doped manganites, for instance, is described as the alternation of $\mathrm{Mn}^{4+}$ and $\mathrm{Mn}^{3+}$ species $[18,19]$; however, the degree of charge disproportionation is much smaller and is better described by $\mathrm{Mn}$ valences of $3.5+\delta\left(\mathrm{Mn}^{4+}\right.$-like $)$ and $3.5-\delta\left(\mathrm{Mn}^{3+}\right.$-like $)$ with $\delta \ll 0.5$ [20, 29]. In reality, the crystal structure also undergoes a variety of complex atomic displacements, such as Jahn-Teller and breathing distortions, which alter the bonding network and hence the electronic configuration. Therefore, emergent charge and orbital textures are closely linked to the pattern and symmetry of said displacements [30, 31]. For site-centered order, bulk X-ray or neutron structural data suggests that the crystal adopts $P 2_{1} / m$ space group symmetry with bond distortions consistent with charge localization on the Mn sites [19, 20, 22]. The experimental report for the bond-centered model, on the other hand, found a different space group symmetry $\left(P n m 2_{1}\right)$ and a distinct displacement pattern associated with it [21]. The ability to probe intra-unit-cell lattice distortions can therefore determine or even reveal novel ground states.

To firmly connect patterns of atomic displacements to the reported crystal symmetries and hence to the models of electronic order, we first explore the possible distorted structures that emerge from the high-symmetry phase (space group Pnma) using group theory. Any distorted structure must double the unit cell, therefore we require atomic displacements whose wavevector is $\mathbf{q}=(1 / 2,0,0)$. The relevant distortion consistent with this requirement is the $\mathrm{X} 1$ displacement mode, or irreducible representation (irrep), which affects two inequivalent Mn sublattices in the high-symmetry unit cell (See Methods section 4). In other words, the X1 irrep is two-dimensional with the first dimension corresponding to the first Mn sublattice (Mn-1 in blue) and the second dimension to the second Mn sublattice (Mn-2 in red), as shown in Fig. 2a. If X1 displacements, which consist of a complex set of atomic distortions including the transverse Mn displacements shown in Fig. 2, affect only the first sublattice, the resulting crystal structure has $P 2_{1} / m$ symmetry, which is consistent with site-centered order (Fig. 2b,c). If they occur in both sublattices and with equal magnitude (Fig. 2b,d), the resulting crystal structure has Pnm $2_{1}$ symmetry, which matches that of bond-centered order. A third structure with Pm symmetry can be obtained by having displacements on both sublattices but with different magnitude (Fig. 2b,e). Such an intermediate state combines aspects of both site and bond order, however, experimen- 


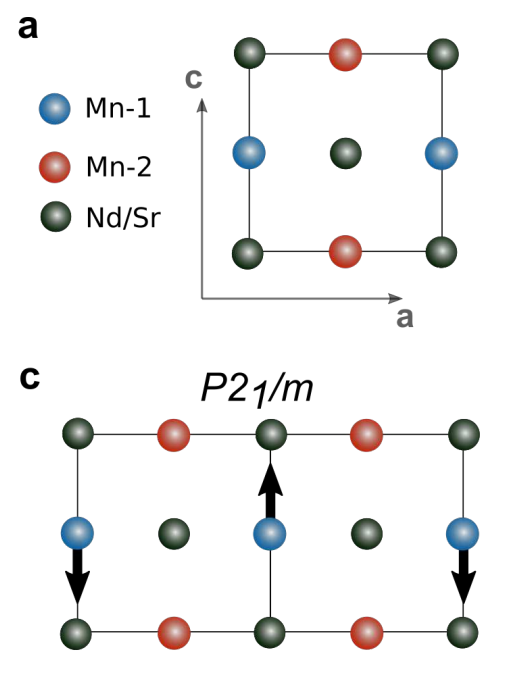

a
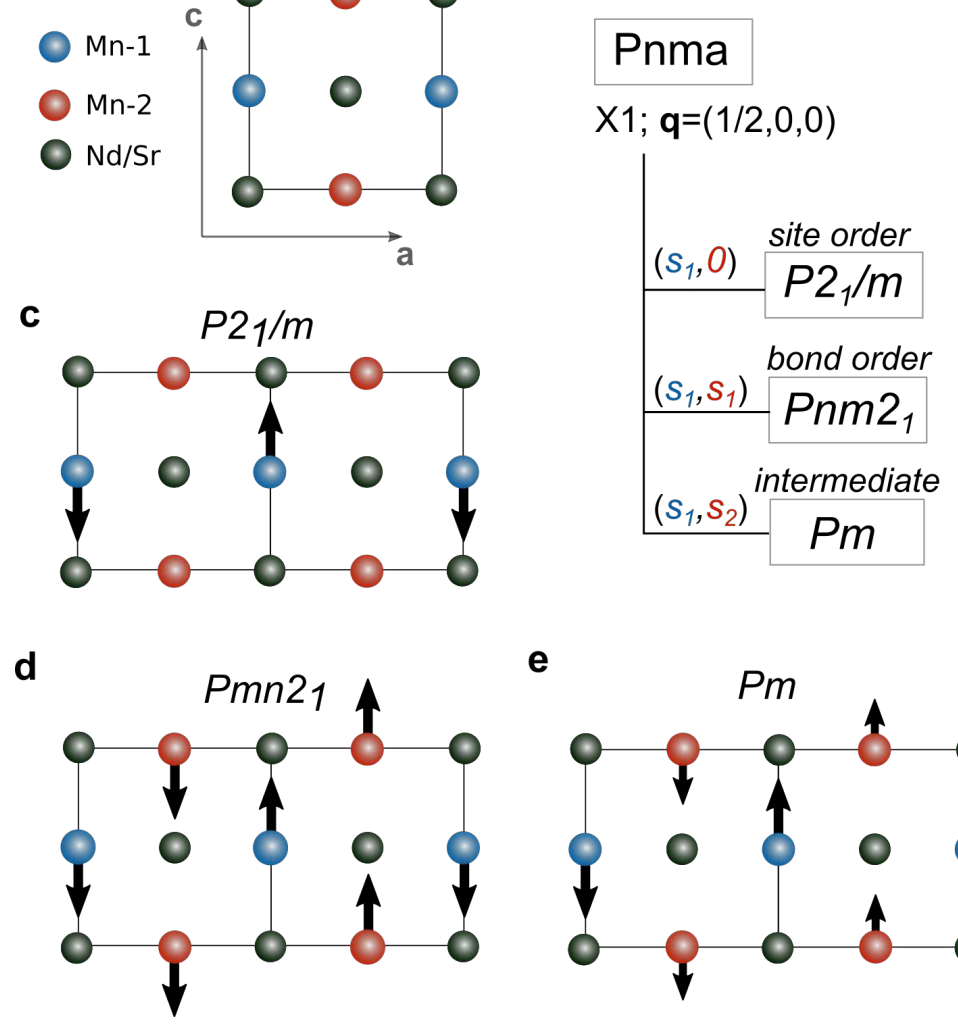

e

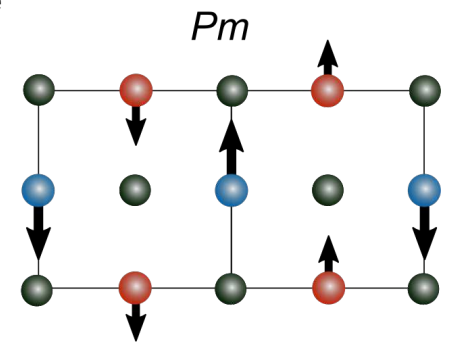

FIG. 2. The symmetry of distorted structures. a, High-symmetry unit cell with two Mn sublattices. b, Group-subgroup relations for displacements with the wavevector $\mathbf{q}=(1 / 2,0,0)$ and irreducible representation $\mathrm{X} 1$. The $\mathrm{X} 1$ order parameter is two dimensional with each dimension corresponding to a Mn sublattice. c-e, Displacement patterns for the site-centered $\left(s_{1}, s_{2}=0\right)$, bond-centered $\left(s_{1}, s_{2}=s_{1}\right)$, and intermediate $\left(s_{1}, s_{2} \neq s_{1}\right)$ phase, respectively.

tal refinements of atomic positions in this symmetry have not been performed so far. The group theory analysis thus shows that the pattern of Mn displacements uniquely defines the character of the charge-ordered phase, without resorting to measurements of the electronic charge.

We now pinpoint the underlying ordering model in $\mathrm{Nd}_{1 / 2} \mathrm{Sr}_{1 / 2} \mathrm{MnO}_{3}$, by mapping the picoscale lattice degrees of freedom using HAADF-STEM at low temperature. A reference image lacking the periodic modulation is generated by removing the contribution of the $\mathbf{Q}^{C O}$ superlattice peaks in the Fourier transform [32, 33]. The displacements are then extracted by mapping the atomic positions in the original image and the reference image. Figure 3a shows a STEM image overlaid with arrows indicating Mn column displacements. The area 
of the triangle denotes the magnitude of displacement and the color represents the angle of the displacement relative to the wavevector. The dominant displacements have transverse polarization and generate a twofold superlattice.

The displacement pattern shown in Fig. 3a matches site-centered order. The first Mn sublattice has a large displacement amplitude (8.2(9) pm) while the second has a comparatively negligible amplitude (1.3(6) pm), similar to the group theory prediction of site-centered order. The manganese displacements as a function of position, $\mathbf{r}$, are sinusoidal (Fig. 3c) and may be described by $\boldsymbol{\Delta}_{\mathrm{Mn}}(\mathbf{r})=\mathbf{A} \sin \left(\mathbf{Q}^{C O} \cdot \mathbf{r}+\phi\right)$ where $\boldsymbol{\Delta}_{\mathrm{Mn}}(\mathbf{r}), \mathbf{A}, \mathbf{Q}^{C O}$, and $\phi$ are the displacement, amplitude and wavevectors, and the phase, respectively. The phase determines the centering of the modulation relative to the Mn sites, with site- and bond-centered order corresponding to $\phi=n \pi$ ( $n$ is an integer) and $\phi=n \pi / 2$ ( $n$ is an odd integer), respectively. A deviation of the phase from these two limits corresponds to a state intermediate between site and bond order. Fitting the displacements in the region shown in Fig. 3a further supports that the modulation is predominantly site-centered with $\phi=0.04(1) \pi$. From this measurement, we thus confirm the presence of the prototypical site-centered state.

Remarkably, we discover that within the same sample another region has a distinct displacement pattern: prominent Mn displacements occur in both Mn sublattices (Fig. 3b). Importantly, the displacements have different amplitudes on each sublattice, unlike the predicted pattern for purely bond-centered order. By comparing to the group theory analysis of displacement patterns, the observed structure is consistent with a state which is intermediate between pure site and bond order ( $P m$ structure). Figure 3d shows the aggregated Mn displacements and their sinusoidal envelope. The intermediate structure is readily apparent since the maximum of the sinusoidal function is not centered on Mn sites nor exactly at the middle. The phase is $\phi=0.35(1) \pi$ and the mean displacement amplitudes on the first and second sublattices are 2.9(9) pm and 5.8(7) pm, respectively. Given that STEM is a projection imaging technique, we ruled out the possibility that the intermediate state merely reflects a projection of stacked site-centered states along the beam direction. This is achieved by analyzing both the contrast variations in the image and the patterns of displacements (see Methods section 3 for more details). Cryo-STEM mapping of lattice displacements therefore provides direct evidence for intermediate charge order in manganites, an observation with implications for other oxides in which the exact character of charge ordering remains unresolved. 
a
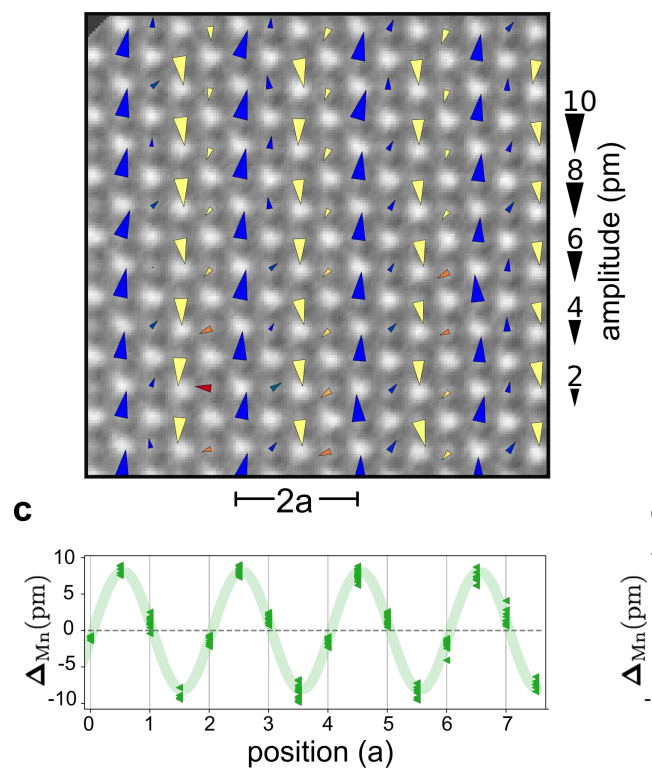

b
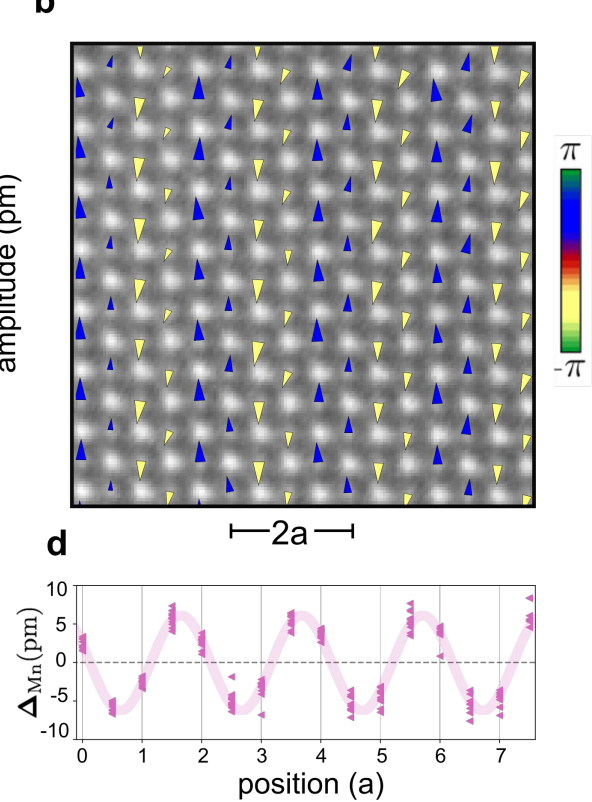

e

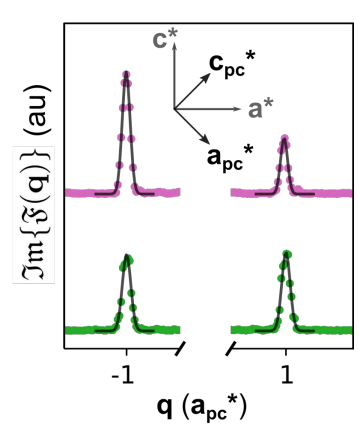

$\mathbf{f}$

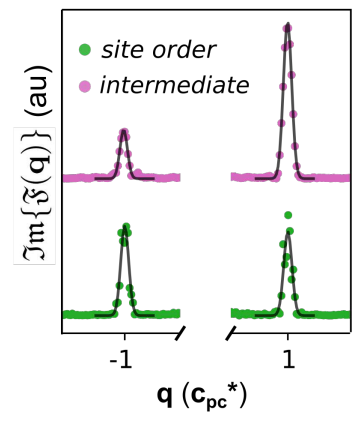

g

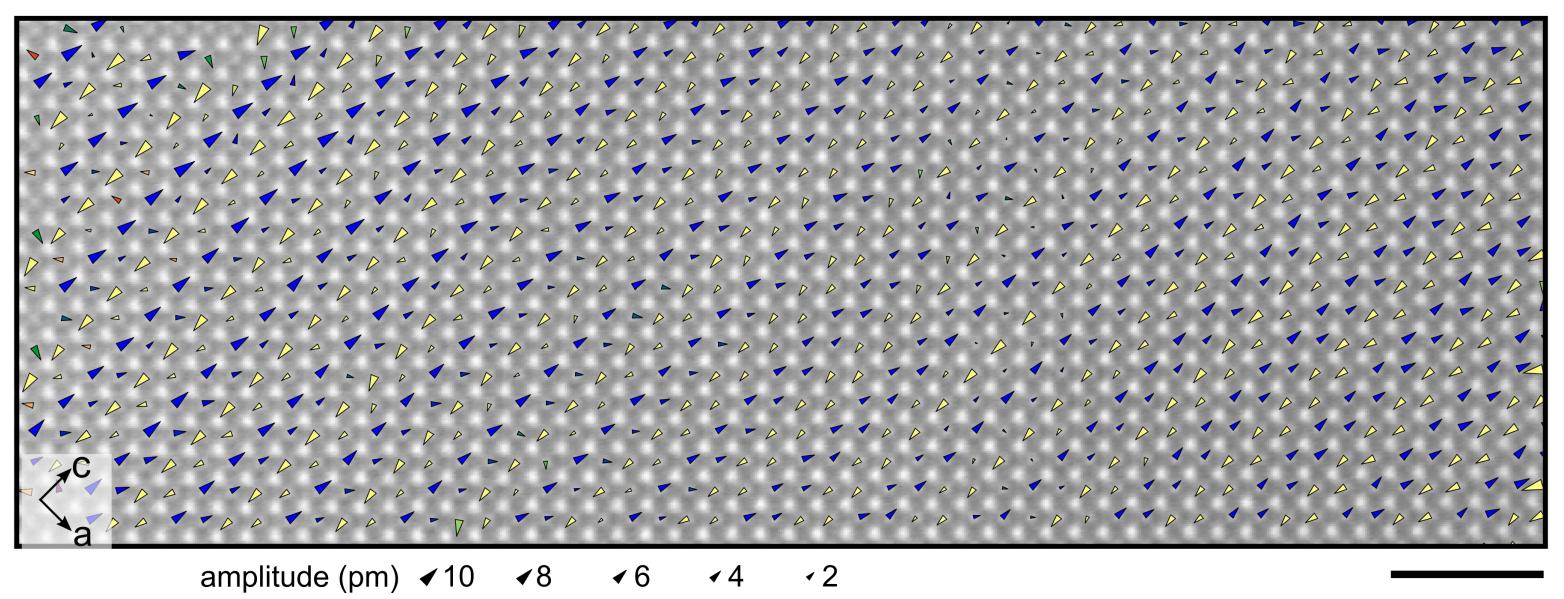

FIG. 3. Nanoscale coexistence of site order and non-centrosymmetric intermediate

order. a, Map of periodic lattice displacements of manganese atomic columns $\left(\boldsymbol{\Delta}_{\mathrm{Mn}}\right)$. The area of the arrows scales with the amplitude of the distortion and the color represents the transverse polarization of the displacements. The displacement pattern within the sub-region is consistent with site-centered order; one Mn sublattice shows large displacement amplitude while the other shows much smaller displacements. b, Periodic lattice displacement map in another sub-region shows displacements on both sublattices. Further, the sublattice displacement amplitudes are not equal which indicates intermediate order. c, Mn displacements (dots) and sinusoidal envelope function (line) in the site-centered phase. $\mathbf{d}$, Mn displacements (dots) and sinusoidal envelope function (line) in the intermediate phase. 
FIG. 3. (continued) e,f, Linecuts across the imaginary part of local Fourier transforms, $\mathfrak{I m}\{\mathfrak{F}(\mathbf{q})\}$, along the pseudocubic directions $\mathbf{a}_{\mathbf{p c}}^{*}$ and $\mathbf{c}_{\mathbf{p c}}^{*}$. The site-centered phase (green) maintains $\pm \mathbf{q}$ symmetry whereas the intermediate phase (magenta) shows clear differences between the $\pm \mathbf{q}$ peaks, an indication that the latter phase breaks inversion symmetry. g, Large field-of-view map of periodic Mn displacements shows a transition from site-centered order (left side) to intermediate order (right side). The scale bar corresponds to $2 \mathrm{~nm}$.

An intriguing consequence of the overlap of site- and bond-centered order is that additional crystal symmetries may be broken [24]. In oxides or charge-transfer salts, intermediate order is predicted to break inversion symmetry due to the formation of uncompensated dipoles, motivating proposals for unconventional ferroelectricity emerging from electronic order [24, 25, 34-37]. To determine whether the intermediate phase is indeed noncentrosymmetric, we compute local complex-valued Fourier transforms of the site-centered and intermediate regions. The imaginary part, $\mathfrak{I m}\{\mathfrak{F}(\mathbf{q})\}$, is sensitive to the odd component of the atomic resolution image and hence to the breaking of inversion symmetry [38]. For a non-centrosymmetric structure, we therefore expect the crystalline Bragg peaks at $\pm \mathbf{q}$ positions to be inequivalent. Figures 3 e and f show integrated line cuts through $\mathfrak{I m}\{\mathfrak{F}(\mathbf{q})\}$ along the pseudocubic $\mathbf{a}_{p c}^{*}$ and $\mathbf{c}_{p c}^{*}$ directions, respectively. In the site-centered phase (green), the imaginary component of the Bragg peaks has the same amplitude at $\pm \mathbf{q}$ coordinates for both pseudo-cubic directions, which indicates that inversion symmetry is maintained. In contrast, there is a significant difference in the intermediate case (magenta), confirming that inversion symmetry is broken in that region. The group theory analysis also tells a consistent story with the intermediate phase having a non-centrosymmetric $(P m)$ structure. While intermediate charge order can in principle be detected though the switching of bulk polarization, the finite conductivity and nanoscale spatial inhomogeneity of manganites have precluded such an approach [25]. The atomic-scale mapping of intermediate order and the detection of the resultant broken inversion symmetry represent a new approach to probe electronically driven symmetry breaking.

Another key insight from these local visualizations is that ground states with distinct symmetries may coexist within the same system. Both Figs. 3A and B are taken from the same field of view image (Fig. 3g), in which the site-centered phase (left side) transforms into 
the intermediate phase (right side) over a few unit cells. This suggests that the energies of site and intermediate orders are comparable and likely linked to subtle spatial fluctuations inherent to strongly interacting oxide materials [27]. Such nanoscale coexistence further highlights the long-standing difficulty in determining the correct charge order model from bulk-averaged measurements, a situation exacerbated by the presence of crystalline twins (see for example Supplementary Fig. 3) and other inhomogeneity.

Informed by the atomic-scale evidence for site and intermediate orders, we next examine the possible origin of these two states. While we have focused on the X1 displacements so far, the low temperature phase may also contain additional structural responses that are allowed by symmetry (Fig. 4a). In particular, a $\Gamma_{4}^{+}$mode, which appears as an oxygen breathing distortion, and a $\Gamma_{2}^{-}$mode, which appears as antipolar displacements of Mn atoms, are such symmetry-allowed responses (see Methods section 4). To understand their role we expand the Landau free energy about the high-symmetry phase up to fourth order in powers of the $\Gamma_{4}^{+}, \Gamma_{2}^{-}$, and X1 displacement mode amplitudes (see equation (3) in Methods section 5 for the expansion). Significantly, this expansion contains two relevant third-order coupling terms:

$$
F_{3}=\delta_{b s s} Q_{B}\left(s_{1}^{2}-s_{2}^{2}\right)+\delta_{t s s} Q_{A P} s_{1} s_{2}
$$

where $Q_{B}, Q_{A P}$, and $\left(s_{1}, s_{2}\right)$ are the amplitudes of the $\Gamma_{4}^{+}, \Gamma_{2}^{-}$, and X1 distortions, respectively. From density functional theory (DFT) calculations (see Methods section 5), we find that the $\delta_{t s s}$ and $\delta_{b s s}$ coefficients are negative, which indicates that the coupling terms lower the energy. We therefore propose that the third-order nonlinear couplings between the $\Gamma$ and X1 modes provide a mechanism for favoring one ground state over another.

We now consider the implications of the two coupling terms in equation (1). The first term stabilizes the breathing distortion and lowers the energy the most in the site-centered phase where the X1 displacements are $\left(s_{1} \neq 0, s_{2}=0\right)$. In contrast, the second term lowers the energy the most in the presence of the antipolar distortions and the bond-centered phase which has X1 displacements $\left(s_{1}, s_{2}=s_{1}\right)$. Both coupling terms will contribute to lowering the energy in the intermediate case where the $\mathrm{X} 1$ amplitude is $\left(s_{1}, s_{2} \neq s_{1}\right)$. To illustrate, we show one-dimensional line cuts across the minimum of the Landau energy in Fig. 4b, having set the coefficients and amplitudes to the DFT-calculated values (see Methods section 5 and Supplementary Tables II and III). If we keep the first coupling term and suppress the second, the free-energy is minimized by having $\left(s_{1} \neq 0, s_{2}=0\right)$ in 
a
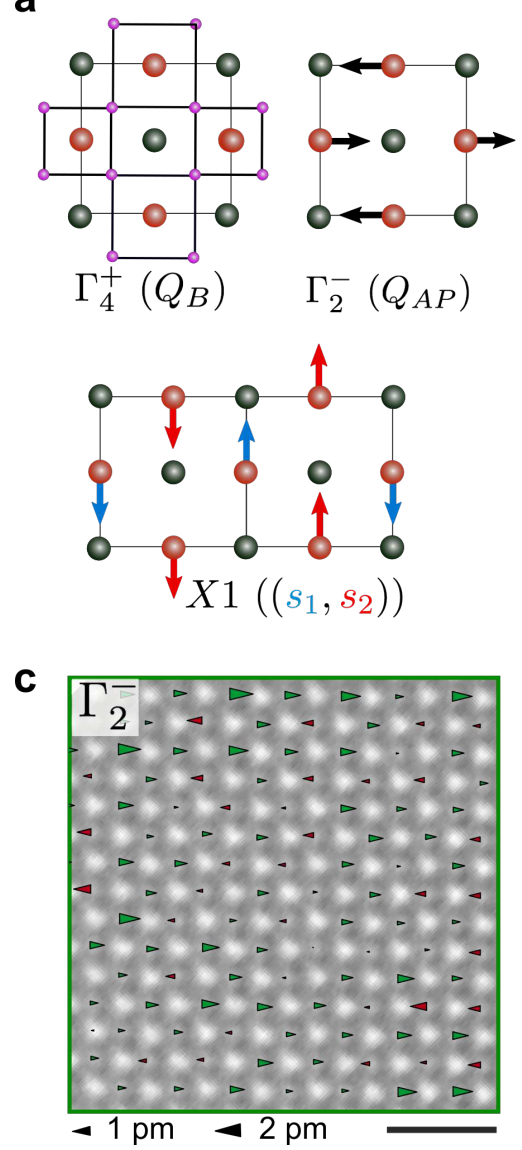

b

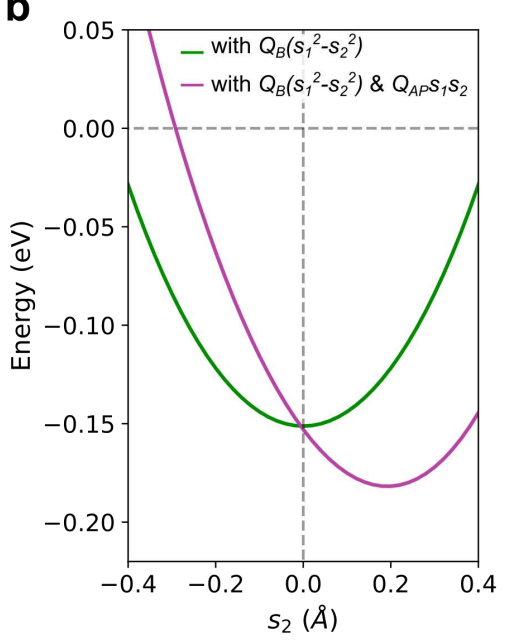

d

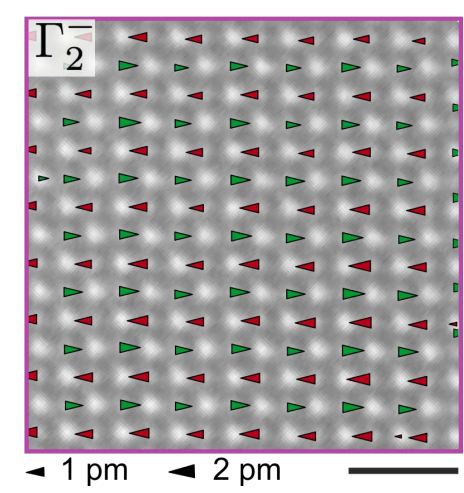

FIG. 4. Non-linear lattice coupling determines the charge order ground state. a, Displacement patterns for the $\Gamma_{4}^{+}$mode (oxygen breathing distortion), the $\Gamma_{2}^{-}$mode (Mn antipolar displacement), and the X1 mode. The purple dots represent the oxygen atoms. b, One dimensional plot of the full Landau free-energy (equation (3) in Methods section 5) along the $\left(0, s_{2}\right)$ direction of X1. When the first coupling term is included and the second term is suppressed (green), the minimum energy is located at $\left(s_{1} \neq 0, s_{2}=0\right)$, indicating that site-centered order is favorable. If both coupling terms are present (magenta), both $s_{1}$ and $s_{2}$ are finite but not equal, indicating that intermediate order is favorable. The values for the coefficients in the Landau free energy and the $Q_{A P} / Q_{B}$ amplitudes are listed in the Methods section 5. The amplitude of $s_{2}$ (in $\AA$ ) is with respect to a 40-atom DFT supercell. c,d, Atomic-scale maps of the $\Gamma_{2}^{-} \mathrm{Mn}$ displacements in the site-centered and intermediate region, respectively. The largest arrows correspond to $\sim 2 \mathrm{pm}$. The displacements are disordered in the former and coherent in the latter, in agreement with the Landau theory predicting that the $\Gamma_{2}^{-}$mode favors X1 displacements on both sublattices. Both scale bars correspond to $1 \mathrm{~nm}$. 
the X1 amplitude, indicating that site-centered order is favorable. When both coupling terms are present, the minimum of the free-energy shifts away from $\left(s_{1}, 0\right)$ to $\left(s_{1}, s_{2} \neq 0\right)$, thus stabilizing the intermediate phase through a non-linear mechanism (see Supplementary Fig. 10 for alternative 2D plots of the energy surfaces). Based on this theory, a key prediction is that the antipolar $\Gamma_{2}^{-}$displacements are absent (present) in the experimentally observed site-centered (intermediate) phase.

To test this prediction, we visualize the spatial interplay between the antipolar Mn displacements and the character of the charge-ordered ground state. Figures 4c and d show maps of the antipolar $\Gamma_{2}^{-}$Mn displacements in the regions containing site and intermediate order, respectively. In the region with site-centered order, the $\Gamma_{2}^{-}$mode is disordered, lacking any clear pattern of antipolar distortions. In the region with intermediate order, however, this mode is coherent and relatively strong, in agreement with the Landau theory prediction.

The unusual non-linear third-order lattice couplings not only challenge our microscopic understanding of the origin of charge ordering but also provide a mechanism for manipulating the character of electronic order and its associated electronic properties. In the case of the non-centrosymmetric intermediate phase, an exciting prospect is to demonstrate ferroelectricity through the switching of polarization; however, the finite conductivity of manganites and the manifestation of the intermediate phase only in localized regions are significant hurdles. To address the latter limitation, one approach inspired by our observations is to enhance the $Q_{A P}$ amplitude which in turn would favor the polar intermediate phase over macroscopic volumes. Such manipulation could be achieved via elastic strain and rare-earth substitution which were found to modulate the amplitude of $\Gamma_{2}^{-}$distortions in related theoretical calculations [39].

Our study shows that cryogenic STEM is an emerging methodology for probing complex electronic ordering phenomena through the important but often neglected lens of the lattice degrees of freedom. Using this approach, we discovered the co-existence of distinct charge order configurations including a pure site-centered phase and a more exotic intermediate phase which breaks inversion symmetry. We also revealed that the stability of these different configurations depends on unique lattice couplings. Such a rich electronic and structural landscape should be relevant to other charge-ordered systems, including cuprates and nickelates where the exact microscopic arrangement associated with various electronic instabilities remains under intense scrutiny. Similar to the current study, novel insights may 
be achieved through direct, real space visualizations that can spatially disentangle different ordering models and characterize the intra-unit-cell structure and symmetry in detail.

[1] Tranquada, J., Sternlieb, B., Axe, J., Nakamura, Y., Uchida, S. Evidence for stripe correlations of spins and holes in copper oxide superconductors. Nature 375, 561 (1995).

[2] Mori, S., Chen, C., Cheong, S.-W. Pairing of charge-ordered stripes in (La,Ca) $\mathrm{MnO}_{3}$. Nature 392, 473 (1998).

[3] Abbamonte, P., Blumberg, G., Rusydi, A., Gozar, A., Evans, P., Siegrist, T., Venema, L., Eisaki, H., Isaacs, E., Sawatzky, G. Crystallization of charge holes in the spin ladder of $\mathrm{Sr}_{14} \mathrm{Cu}_{24} \mathrm{O}_{41}$. Nature 431, 1078-1081 (2004).

[4] Sasaki, S., Hashimoto, K., Kobayashi, R., Itoh, K., Iguchi, S., Nishio, Y., Ikemoto, Y., Moriwaki, T., Yoneyama, N., Watanabe, M., Ueda, A., Mori, H., Kobayashi, K., Kumai, R., Murakami, Y., Müller, J., Sasaki, T. Crystallization and vitrification of electrons in a glassforming charge liquid. Science 357, 1381-1385 (2017).

[5] Chang, J., Blackburn, E., Holmes, A., Christensen, N. B., Larsen, J., Mesot, J., Liang, R., Bonn, D., Hardy, W., Watenphul, A., Zimmermann, M. v., Forgan, E. M., Hayden, S. M. Direct observation of competition between superconductivity and charge density wave order in $\mathrm{YBa}_{2} \mathrm{Cu}_{3} \mathrm{O}_{6.67}$. Nature Physics 8, 871-876 (2012).

[6] Li, L., O'Farrell, E., Loh, K., Eda, G., Özyilmaz, B., Neto, A. C. Controlling many-body states by the electric-field effect in a two-dimensional material. Nature 529, 185-189 (2016).

[7] Gao, M., Lu, C., Jean-Ruel, H., Liu, L. C., Marx, A., Onda, K., Koshihara, S.-y., Nakano, Y., Shao, X., Hiramatsu, T., , et al. Mapping molecular motions leading to charge delocalization with ultrabright electrons. Nature 496, 343-346 (2013).

[8] Milward, G., Calderon, M., Littlewood, P. Electronically soft phases in manganites. Nature 433, 607-610 (2005).

[9] Comin, R., Sutarto, R., daSilva Neto, E., Chauviere, L., Liang, R., Hardy, W., Bonn, D., He, F., Sawatzky, G., Damascelli, A. Broken translational and rotational symmetry via charge stripe order in underdoped $\mathrm{YBa}_{2} \mathrm{Cu}_{3} \mathrm{O}_{6+y}$. Science 347, 1335-1339 (2015).

[10] Comin, R., Sutarto, R., He, F., daSilva Neto, E., Chauviere, L., Frano, A., Liang, R., Hardy, W., Bonn, D., Yoshida, Y., Eisaki, H., Achkar, A. J., Hawthorn, D. G., Keimer, B., Sawatzky, 
G. A., Damascelli, A. Symmetry of charge order in cuprates. Nature Materials 14, 796-800 (2015).

[11] Achkar, A., He, F., Sutarto, R., McMahon, C., Zwiebler, M., Hücker, M., Gu, G., Liang, R., Bonn, D., Hardy, W., Geck, J., Hawthorn, D. G. Orbital symmetry of charge-density-wave order in $\mathrm{La}_{1.875} \mathrm{Ba}_{0.125} \mathrm{CuO}_{4}$ and $\mathrm{YBa}_{2} \mathrm{Cu}_{3} \mathrm{O}_{6.67}$. Nature Materials 15, 616 (2016).

[12] Zhao, H., Ren, Z., Rachmilowitz, B., Schneeloch, J., Zhong, R., Gu, G., Wang, Z., Zeljkovic, I. Charge-stripe crystal phase in an insulating cuprate. Nature Materials 18, 103 (2019).

[13] Tranquada, J., Buttrey, D., Sachan, V., Lorenzo, J. Simultaneous ordering of holes and spins in $\mathrm{La}_{2} \mathrm{NiO}_{4.125}$. Physical Review Letters) 73 (1994).

[14] Uehara, M., Mori, S., Chen, C. H., Cheong, S. W. Percolative phase separation underlies colossal magnetoresistance in mixed-valent manganites. Nature 399, 560-563 (1999).

[15] Khomskii, D. (2014) Transition metal compounds. Cambridge University Press.

[16] McMahon, C., Achkar, A., Neto, E., Djianto, I., Menard, J., He, F., Sutarto, R., Comin, R., Liang, R., Bonn, D., Hardy, W. N., Damascelli, A., Hawthorn, D. G. Orbital symmetries of charge density wave order in $\mathrm{YBa}_{2} \mathrm{Cu}_{3} \mathrm{O}_{6+x}$. arXiv:1904.12929 (2019).

[17] Tokura, Y., Nagaosa, N. Orbital physics in transition-metal oxides. Science 288, 462-468 (2000).

[18] Goodenough, J. B. Theory of the role of covalence in the perovskite-type manganites [La,M(II)]MnO3. Physical Review 100, 564-573 (1955).

[19] Radaelli, P. G., Marezio, M., Cheong, S.-W. Charge, orbital, and magnetic ordering in $\mathrm{La}_{0.5} \mathrm{Ca}_{0.5} \mathrm{MnO}_{3}$. Physical Review B 55, 3015-3023 (1997).

[20] Goff, R., Attfield, J. Charge ordering in half-doped manganites. Physical Review B 70, 140404 (2004).

[21] Daoud-Aladine, A., Rodriguez-Carvajal, J., Pinsard-Gaudart, L., Fernandez-Diaz, M., Revcolevschi, A. Zener polaron ordering in half-doped manganites. Physical Review Letters 89, 097205 (2002).

[22] Rodriguez, E. E., Proffen, T., Llobet, A., Rhyne, J. J., Mitchell, J. F. Neutron diffraction study of average and local structure in $\mathrm{La}_{0.5} \mathrm{Ca}_{0.5} \mathrm{MnO}_{3}$. Physical Review B 71, 1-9 (2005).

[23] Jooss, C., Wu, L., Beetz, T., Klie, R., Beleggia, M., Schofield, M., Schramm, S., Hoffmann, J., Zhu, Y. Polaron melting and ordering as key mechanisms for colossal resistance effects in manganites. Proceedings of the National Academy of Sciences 104, 13597-13602 (2007). 
[24] Efremov, D. V., Van Den Brink, J., Khomskii, D. I. Bond- Versus site-centred ordering and possible ferroelectricity in manganites. Nature Materials 3, 853-856 (2004).

[25] Van Den Brink, J., Khomskii, D. I. Multiferroicity due to charge ordering. Journal of Physics: Condensed Matter 20, 434217 (2008).

[26] Ogimoto, Y., Nakamura, M., Takubo, N., Tamaru, H., Izumi, M., Miyano, K. Strain-induced crossover of the metal-insulator transition in perovskite manganites. Physical Review B 71, $060403(2005)$.

[27] Lai, K., Nakamura, M., Kundhikanjana, W., Kawasaki, M., Tokura, Y., Kelly, M. A., Shen, Z.-X. Mesoscopic percolating resistance network in a strained manganite thin film. Science 329, 190-193 (2010).

[28] Kuwahara, H., Tomioka, Y., Asamitsu, A., Moritomo, Y., Tokura, Y. A first-order phase transition induced by a magnetic field. Science 270, 961-963 (1995).

[29] Grenier, S., Hill, J. P., Gibbs, D., Thomas, K. J., Zimmermann, M. v., Nelson, C. S., Kiryukhin, V., Tokura, Y., Tomioka, Y., Casa, D., Gog, T., Venkataraman, C. Resonant X-ray diffraction of the magnetoresistant perovskite $\mathrm{Pr}_{0.6} \mathrm{Ca}_{0.4} \mathrm{MnO}_{3}$. Phys. Rev. B, 134419 (2004).

[30] Egami, T., Ishihara, S., Tachiki, M. Lattice effect of strong electron correlation: Implication for ferroelectricity and superconductivity. Science 261, 1307-1310 (1993).

[31] Khalyavin, D. D., Johnson, R. D., Orlandi, F., Radaelli, P. G., Manuel, P., Belik, A. A. Emergent helical texture of electric dipoles. Science 369, 680-684 (2020).

[32] Savitzky, B. H., El Baggari, I., Admasu, A. S., Kim, J., Cheong, S.-W., Hovden, R., Kourkoutis, L. F. Bending and breaking of stripes in a charge ordered manganite. Nature Communications 8, 1883 (2017).

[33] El Baggari, I., Savitzky, B. H., Admasu, A. S., Kim, J., Cheong, S.-W., Hovden, R., Kourkoutis, L. F. Nature and evolution of incommensurate charge order in manganites visualized with cryogenic scanning transmission electron microscopy. Proceedings of the National Academy of Sciences 115, 1445-1450 (2018).

[34] Cheong, S.-W., Mostovoy, M. Multiferroics: a magnetic twist for ferroelectricity. Nature Materials 6, 13-20 (2007).

[35] Lopes, A., Araújo, J., Amaral, V., Correia, J., Tomioka, Y., Tokura, Y. New phase transition in the $\operatorname{Pr}_{1-x} \mathrm{Ca}_{x} \mathrm{MnO}_{3}$ System: Evidence for electrical polarization in charge ordered manganites. 
Physical Review Letters 100, 155702 (2008).

[36] Lunkenheimer, P., Müller, J., Krohns, S., Schrettle, F., Loidl, A., Hartmann, B., Rommel, R., De Souza, M., Hotta, C., Schlueter, J. A., Lang, M. Multiferroicity in an organic chargetransfer salt that is suggestive of electric-dipole-driven magnetism. Nature Materials 11, 755 (2012).

[37] Senn, M. S., Wright, J. P., Attfield, J. P. Charge order and three-site distortions in the verwey structure of magnetite. Nature 481, 173-176 (2012).

[38] Hamidian, M. H., Firmo, I. A., Fujita, K., Mukhopadhyay, S., Orenstein, J. W., Eisaki, H., Uchida, S., Lawler, M. J., Kim, E. A., Davis, J. C. Picometer registration of zinc impurity states in $\mathrm{Bi}_{2} \mathrm{Sr}_{2} \mathrm{CaCu}_{2} \mathrm{O}_{8+\delta}$ for phase determination in intra-unit-cell Fourier transform STM. New Journal of Physics 14, 053017 (2012).

[39] Nowadnick, E. A., He, J., Fennie, C. J. Coupled structural distortions, domains, and control of phase competition in polar $\mathrm{SmBaMn}_{2} \mathrm{O}_{6}$. Physical Review B 100, 195129 (2019).

\section{ACKNOWLEDGMENTS}

This work was primarily support by the Department of Defense Air Force Office of Scientific Research (FA 9550-16-1-0305). I.E.B. and L.F.K. acknowledge partial support by the National Sciences Foundation through the PARADIM Materials Innovation Platform (DMR-1539918).This work made use of the Cornell Center for Materials Research facilities supported through the NSF MRSEC program (DMR-1719875). The FEI Titan Themis 300 was acquired through NSF-MRI-1429155, with additional support from Cornell University, the Weill Institute and the Kavli Institute at Cornell. E.A.N. acknowledges support from the University of California, Merced and the use of computational resources supported by the Cornell University Center for Advanced Computing. The work at Stanford was supported by the the U.S. Department of Energy, Office of Basic Energy Sciences, Division of Materials Sciences and Engineering, under contract no. DE-AC02-76SF00515.

\section{COMPETING INTERESTS}

The authors declare that they have no competing financial interests. 


\section{DATA AVAILABILITY}

The data that support the findings of this study are available from the corresponding author on reasonable request.

\section{CORRESPONDENCE}

Correspondence and requests for materials should be addressed to L.F.K. (email: lena.f.kourkoutis@cornell.edu)

\section{AUTHOR CONTRIBUTIONS}

D.L., Y.H. and H.H. synthesized the samples and performed magnetic characterization. D.J.B, I.E., M.J.Z. and L.F.K. acquired and analyzed the electron microscopy data. E.A.N. performed density functional theory calculations. E.A.N., I.E. and L.F.K. performed symmetry and theoretical analysis. I.E., E.A.N., D.J.B. and L.F.K. wrote the manuscript with input from all authors. 


\section{Figures}

a

\section{site order}

○

C

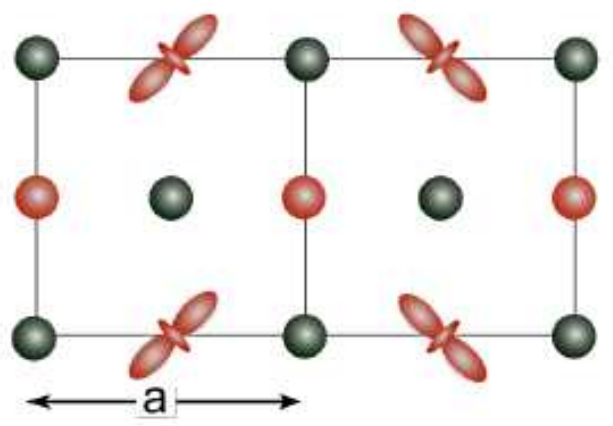

- $\mathrm{Mn}^{4+}$-like 2 \& $\mathrm{Mn}^{3+}$-like

e

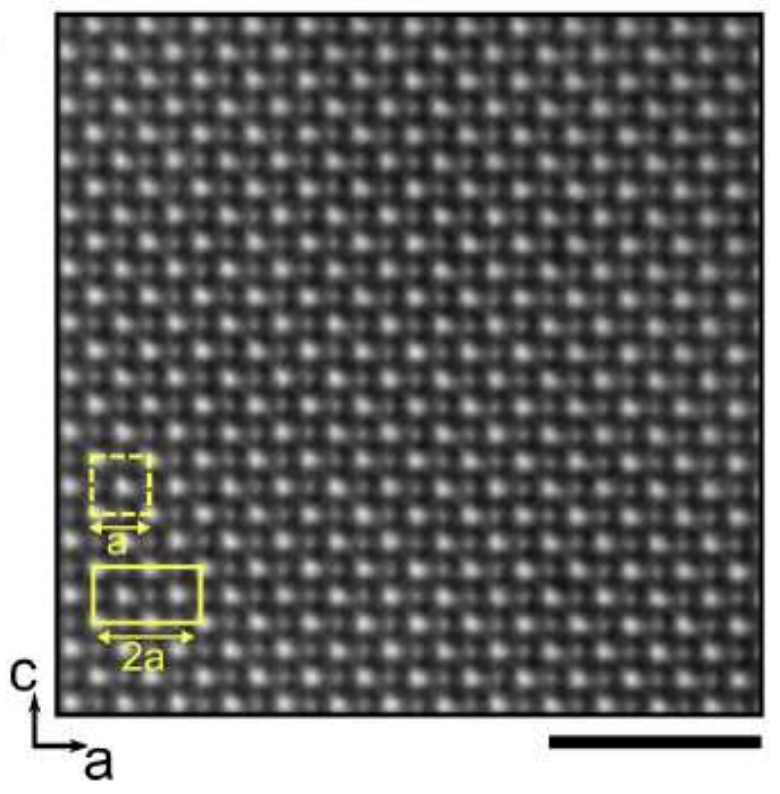

b

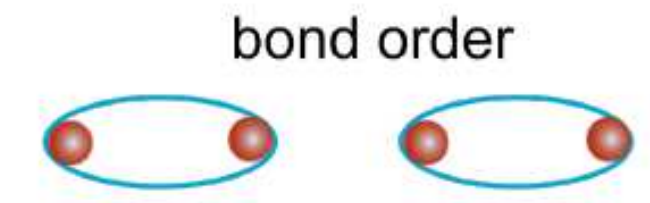

d bond order

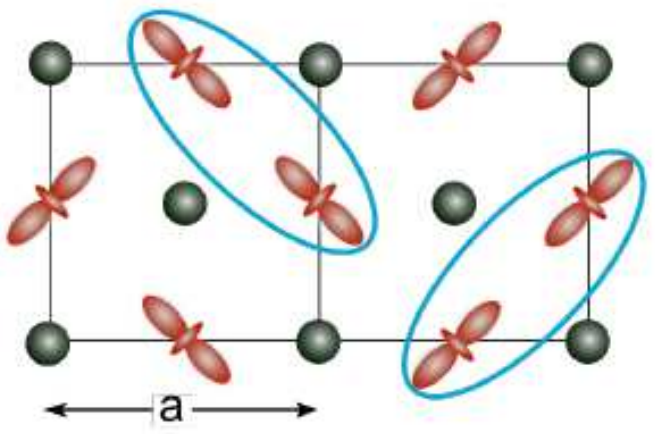

$\mathrm{ONd} / \mathrm{Sr} \quad 28 \mathrm{Mn}^{3.5+}$

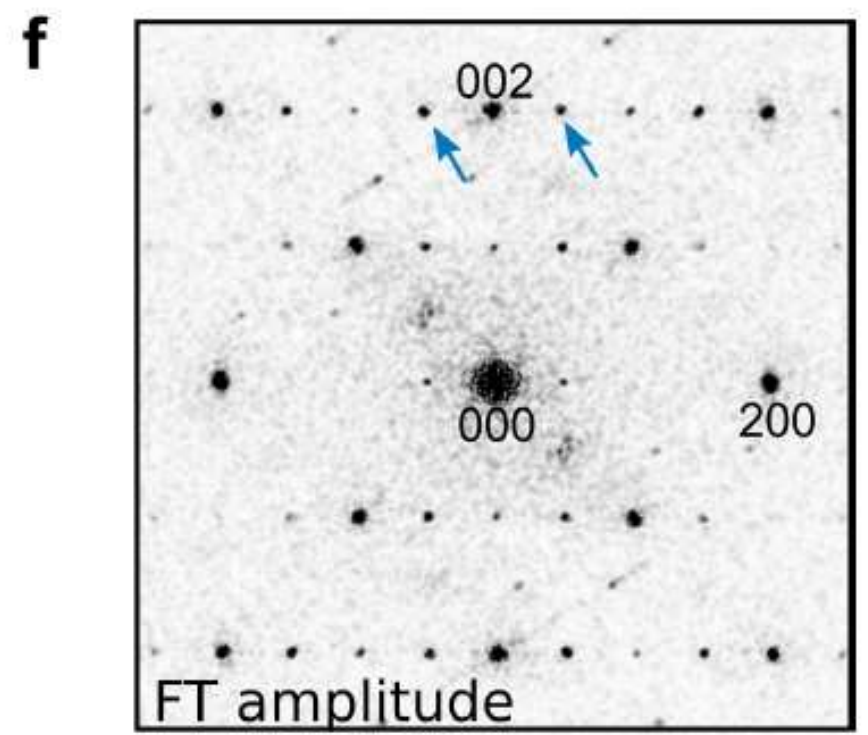

Figure 1

Charge order supercell. a, Charge order modulation centered on sites (red circles). The size of the circle indicates the electronic density on the site. $b$, Charge order modulation centered on the bonds. Ellipses represent stronger, more electron-rich bonds. The sites remain equivalent in density. c, Prototypical sitecentered charge and orbital order model in the half-doped manganites, including orbital order. $d$, Alternative bond-centered model in which all $\mathrm{Mn}$ sites have the same charge configuration (Mn3:5+). In 
this case, the supercell is generated by the orbital order. e, Highangle annular dark-field (HAADF) scanning transmission electron microscopy (STEM) projection image at $93 \mathrm{~K}$, where we expect the system to be in the charge-ordered phase. Bright atomic columns represent $\mathrm{Nd} / \mathrm{Sr}$ sites and dark atomic columns represent $\mathrm{Mn}$ sites. The scale bar corresponds to $2 \mathrm{~nm}$. f, Fourier transform amplitude of the STEM image. At $93 \mathrm{~K}$, superlattice peaks (arrows) with QCO $=(1 / 2,0,0)$ are evident, indicating the formation of a twofold superlattice in real space (box in E).

a

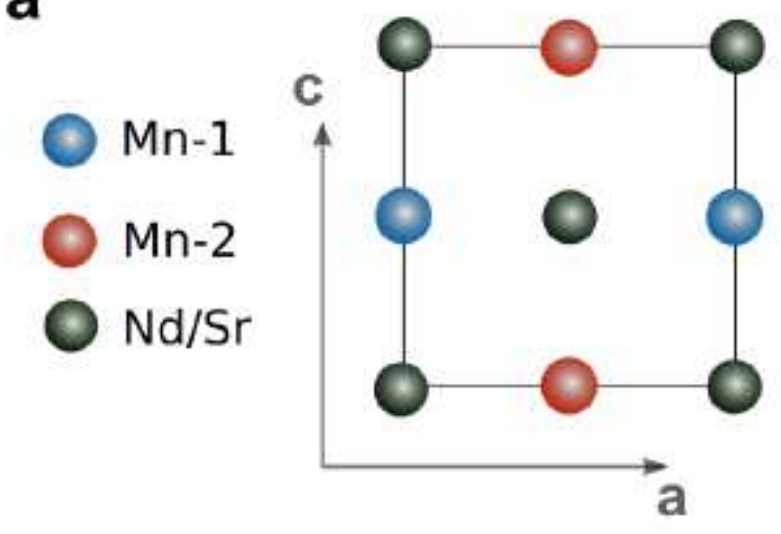

C

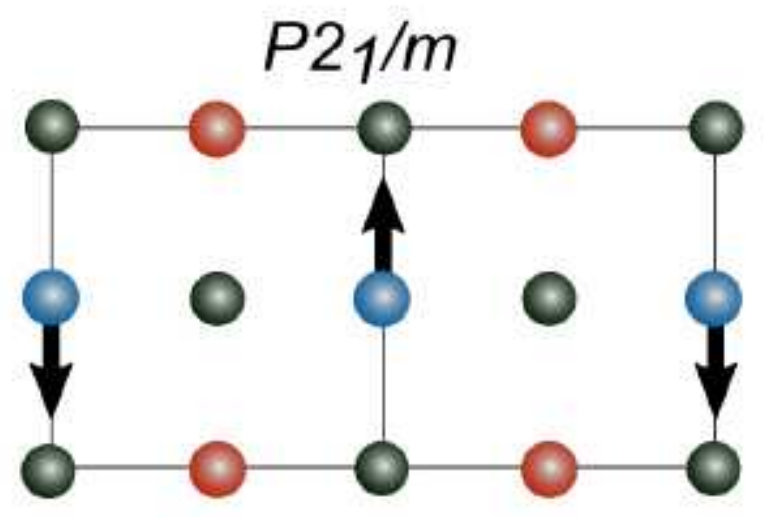

d

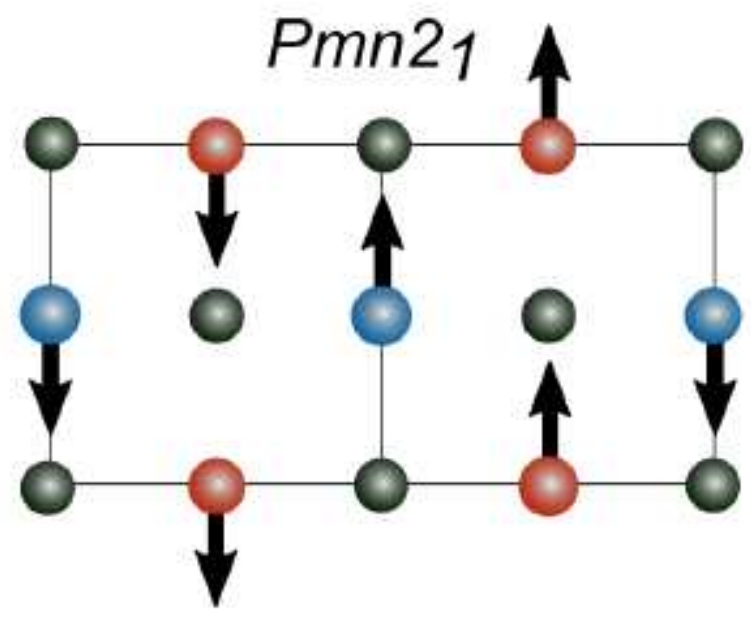

a

\section{Pnma}

$X 1 ; \mathbf{q}=(1 / 2,0,0)$

$\left(s_{1}, 0\right) \quad \begin{gathered}\text { site order } \\ P 2_{1} / m\end{gathered}$

$\left(s_{1}, s_{1}\right)$ bond order

$\left(s_{1}, s_{2}\right)$ intermediate

e

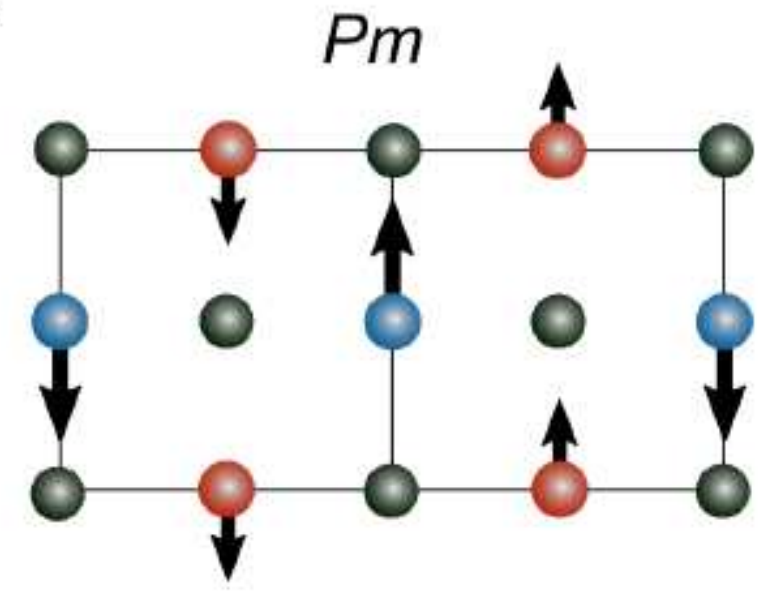

Figure 2 
The symmetry of distorted structures. a, High-symmetry unit cell with two Mn sublattices. b, Groupsubgroup relations for displacements with the wavevector $q=(1=2 ; 0 ; 0)$ and irreducible representation $\mathrm{X} 1$. The $\mathrm{X} 1$ order parameter is two dimensional with each dimension corresponding to a Mn sublattice. Ce, Displacement patterns for the site-centered $(s 1, s 2=0)$, bond-centered $(s 1, s 2=s 1)$, and intermediate $(s 1, s 2 \neq s 1)$ phase, respectively

a
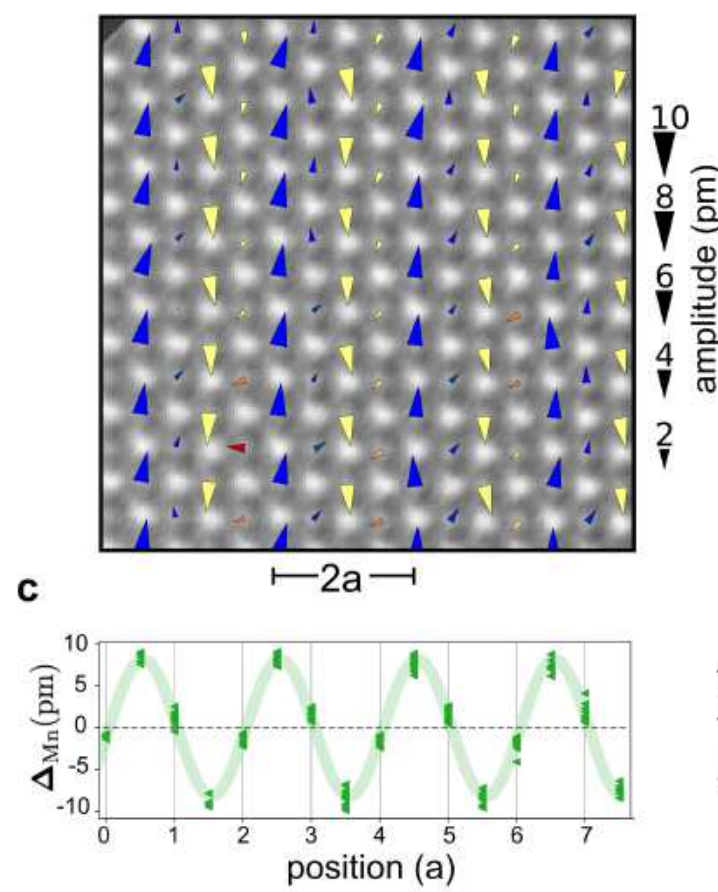

b
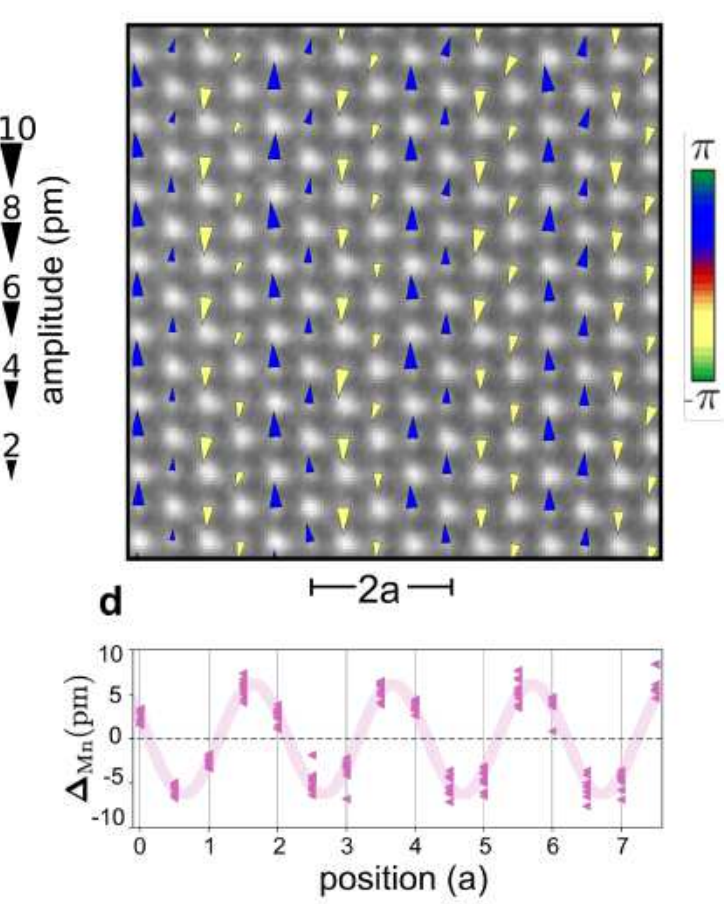

e

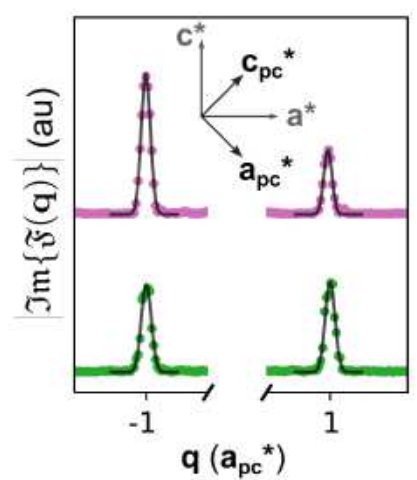

f

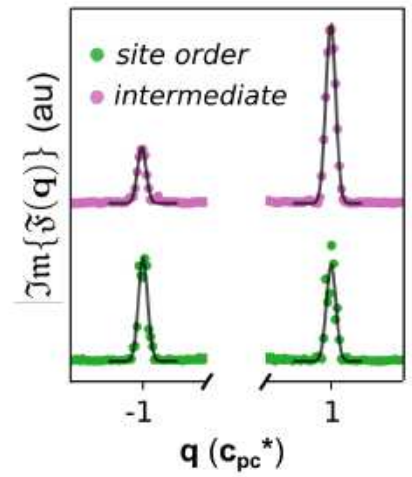

g

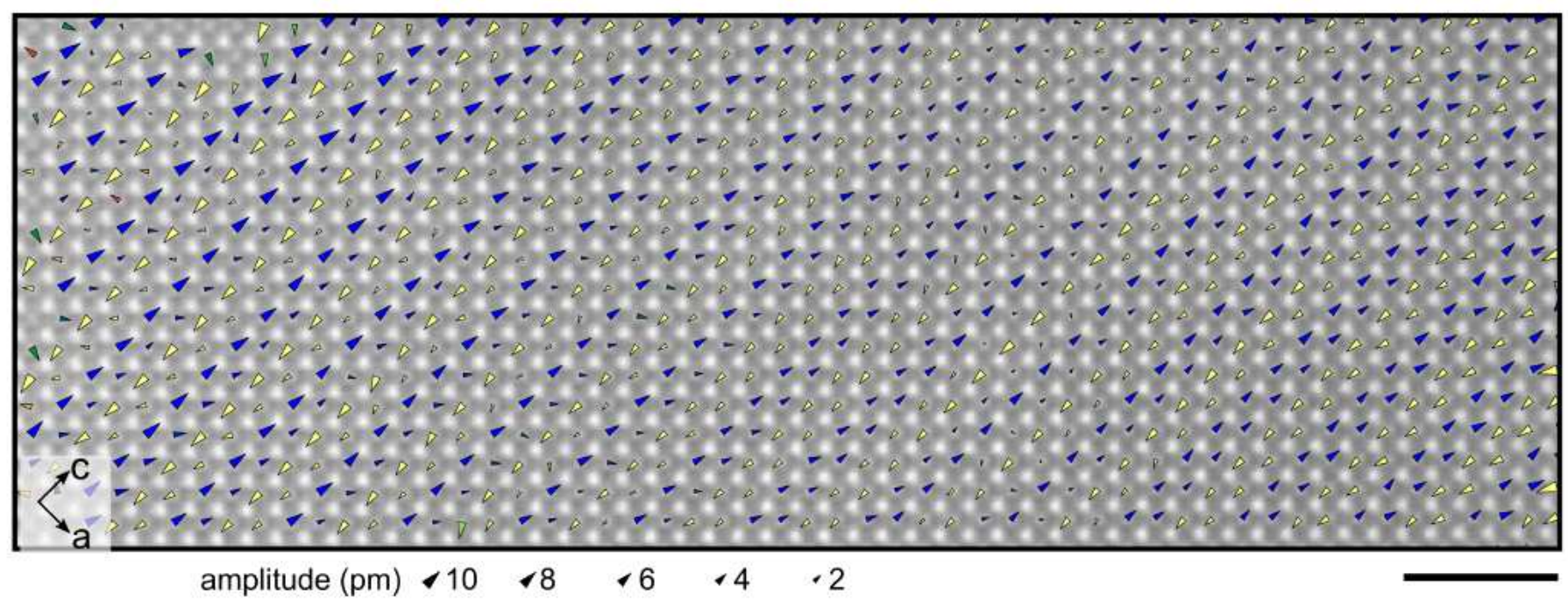

Figure 3

Nanoscale coexistence of site order and non-centrosymmetric intermediate order. a, Map of periodic lattice displacements of manganese atomic columns $(\Delta M n)$. The area of the arrows scales with the amplitude of the distortion and the color represents the transverse polarization of the displacements. The displacement pattern within the sub-region is consistent with site-centered order; one Mn sublattice 
shows large displacement amplitude while the other shows much smaller displacements. b, Periodic lattice displacement map in another sub-region shows displacements on both sublattices. Further, the sublattice displacement amplitudes are not equal which indicates intermediate order. c, Mn displacements (dots) and sinusoidal envelope function (line) in the site-centered phase. $d, \mathrm{Mn}$ displacements (dots) and sinusoidal envelope function (line) in the intermediate phase.

a
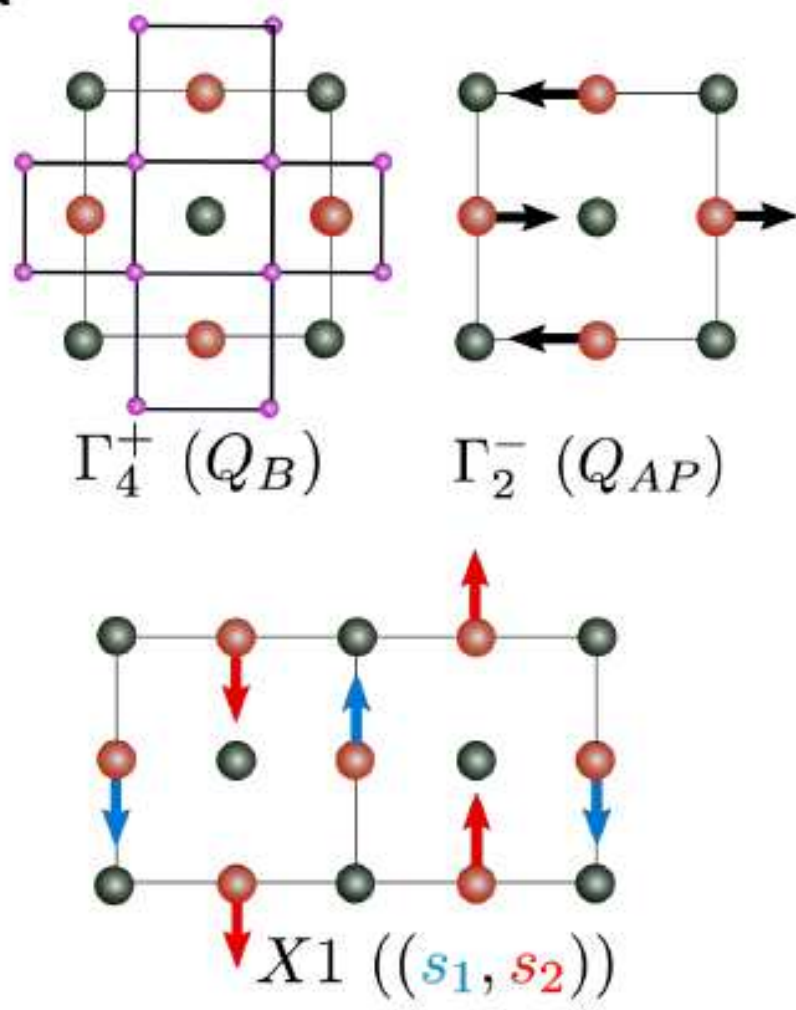

C

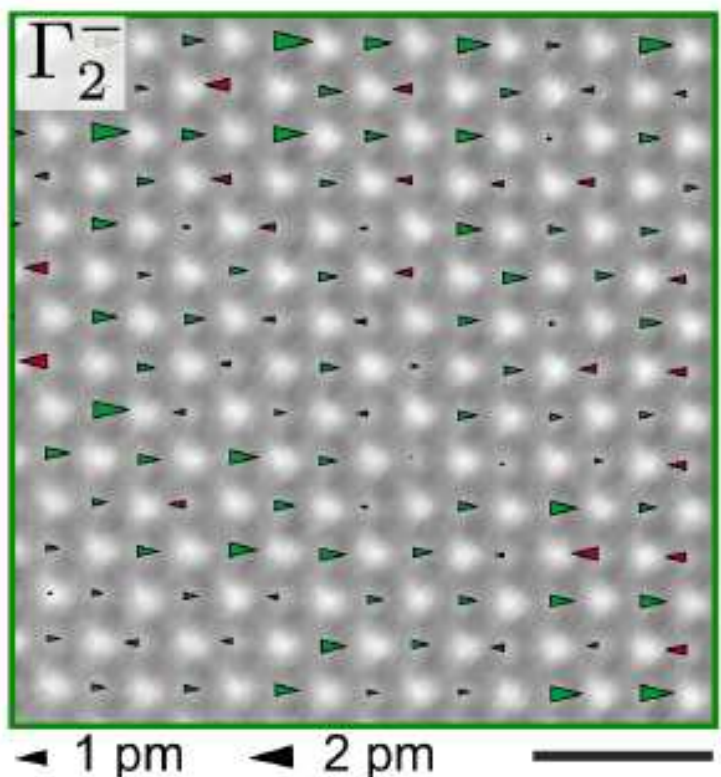

b

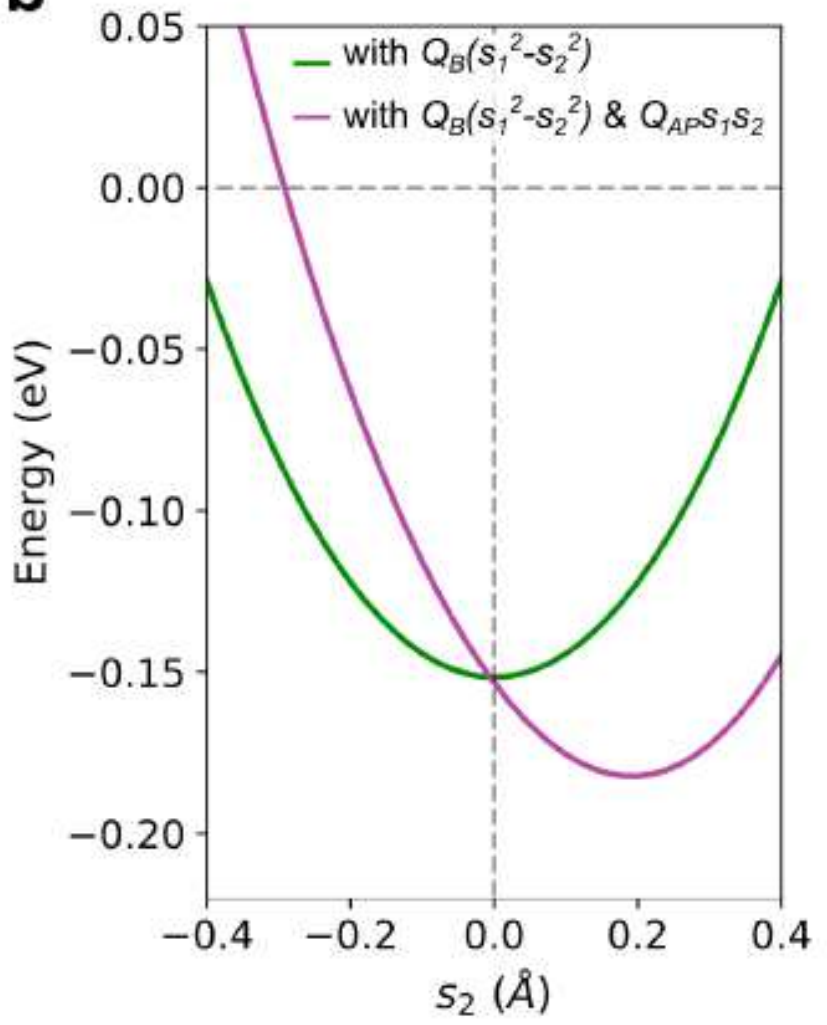

d

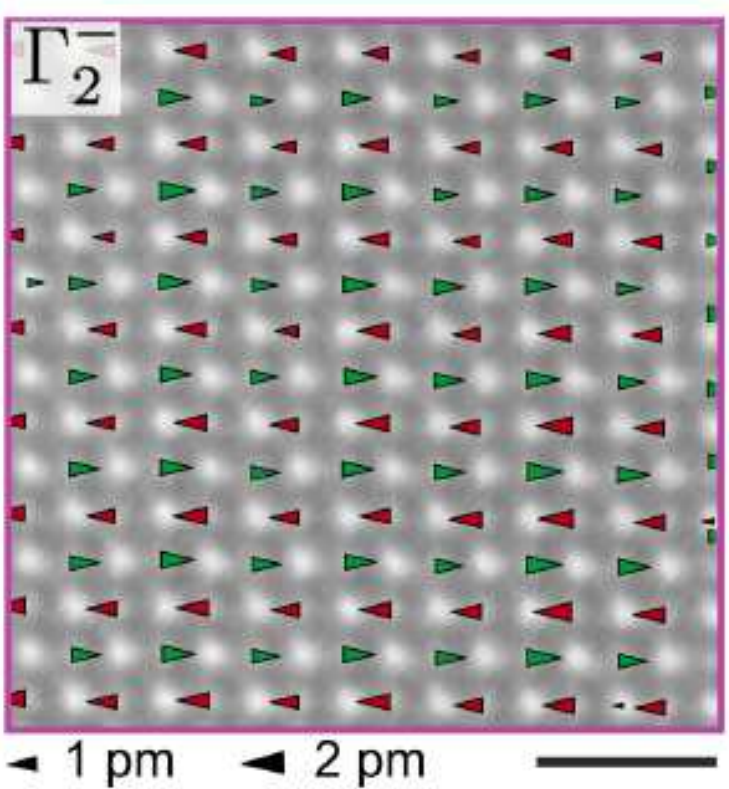

Figure 4 
Non-linear lattice coupling determines the charge order ground state. a, Displacement patterns for the $\Gamma+4$ mode (oxygen breathing distortion), the $\Gamma-2$ mode (Mn antipolar displacement), and the $\mathrm{X} 1$ mode. The purple dots represent the oxygen atoms. $b$, One dimensional plot of the full Landau free-energy (equation (3) in Methods section 5$)$ along the $(0, s 2)$ direction of $X 1$. When the first coupling term is included and the second term is suppressed (green), the minimum energy is located at ( $s 1 \neq 0, \mathrm{~s} 2=0)$, indicating that site-centered order is favorable. If both coupling terms are present (magenta), both s1 and s2 are finite but not equal, indicating that intermediate order is favorable. The values for the coefficients in the Landau free energy and the QAP / QB amplitudes are listed in the Methods section 5 . The amplitude of s2 (in $\AA$ ) is with respect to a 40-atom DFT supercell. c,d, Atomic-scale maps of the $\Gamma-2 \mathrm{Mn}$ displacements in the site-centered and intermediate region, respectively. The largest arrows correspond to $\sim 2 \mathrm{pm}$. The displacements are disordered in the former and coherent in the latter, in agreement with the Landau theory predicting that the $\Gamma-2$ mode favors $X 1$ displacements on both sublattices. Both scale bars correspond to $1 \mathrm{~nm}$.

\section{Supplementary Files}

This is a list of supplementary files associated with this preprint. Click to download.

- methodsSI.pdf 